\section{E0240 EFFECTS OF ATORVASTATIN ON THE EXPRESSIONS OF PECAM-1 AND P-SELECTIN IN ATHEROSCLEROTIC RABBITS}

doi:10.1136/hrt.2010.208967.240

Liao Wei, Cai Jiumei, Li Faquan. The Affiliated Hospital of Gannan Medical College, Jiangxi, China

Objective To investigate the effects of atorvastatin on atherosclerotic rabbits serum lipid concentration and the expressions of PECAM-1 and P-selectin in rabbit thoracic aorta.

Methods Atherosclerotic rabbit models were made by feeding the rabbits with high cholesterol. Thirty New Zealand big rabbits, weight $(2.0 \pm 0.2) \mathrm{kg}$, were randomly divided into three groups. Group A was fed with common forage, Group B was fed with high cholesterol diet, and group $\mathrm{C}$ was fed with high cholesterol diet and atorvastatin simultaneously. After 16 weeks feeding, serum TC, TG, LDL-C, VLDL-C were measured. The aortic arteries were isolated and the samples adjacent to aortic arch were harvested for formicroscopy detection. The PECAM-1 and P-selectin gene expressions in rabbit thoracic aorta were detected by RT-PCR.

Results The levels of TC, TG, LDL-C, VLDL-C in group B were significantly increased compared with group $B$ and group $C(p<0.05)$, while the levels of TC, TG, LDL-C, VLDL-C in group C were higher than those in group $A(p<0.05)$. The aorta structure in group $A$ was clear, the aortic intimal in group B was significantly thick and infiltrated by a large number of foam and inflammatory cells, but the endothelium in group $\mathrm{C}$ only showed focal infiltration of those cells observed under the microscope. The mRNA expression of PECAM-1 and $\mathrm{P}$-selectin in group $\mathrm{B}$ were significantly increased than in group $\mathrm{A}$ $(p<0.01)$, while those in group $C$, which were also higher than group A, were significantly decreased than in group $B(p<0.05)$.

Conclusions Atorvastatin regulates lipid metabolism effectively and reduces the PECAM-1 and P-selectin expression, which are closely related to atherosclerosis.

\section{e0241 EVALUATE SERIATE PROGRESS OF VULNERABLE PLAQUE BY OPTICAL COHERENCE TOMOGRAPHY ON RABBIT VULNERABLE PLAQUE MODEL}

doi:10.1136/hrt.2010.208967.241

Hu Sining, Fang Yan, Liu Haixia, Yang Guang, Tian Jinwei, Jia Haibo, Wu Jian, Meng Lingbo, Hou Jingbo, Yv Bo. Department of Cardiology, Second Affiliated Hospital of Harbin Medical University, Key Laboratories of Education Ministry For Myocardial Ischaemia Mechanism and Treatment, Harbin, Heilongjiang, China

Objectives Assess seriate progress of vulnerable plaque (VP) formation fusing Optical Coherence Tomography (OCT) with histopathological test to confirm what we find in the OCT imaging of vulnerable plaque on established rabbit vulnerable plaque model. Providing early characteristics of the signals displayed by $\mathrm{VP}$ in order to prior recognise vulnerable people, in order to give accurate and reasonable therapy.

Method $23 \mathrm{New}$ Zealand white rabbits were randomly divided into 4 groups after balloon injurying the intima with subsequently $1 \%$ high fat diet. Grouping: Examed at 4 time points: $6(n=6), 8(n=5), 10$ $(\mathrm{n}=5), 12(\mathrm{n}=7)$ weeks. OCT and histopathological examination were performed at those time points to display each stage of vulnerable plaque. Serum blood fat test were collected, especially the low density lipoprotein (LDL-C) as a major predictor for the stability of the plaque. Results There were 2 rabbits died separately in 6 and 8 weeks, and 3 died at 12 weeks. At 6 th week $(n=4)$, OCT showed that the intima were more and more thick with heterogeneously low and high signals. Part of the tissue was protruding into the lumen. The composition of this tissue vertified by pathology is lipid infiltration; At 8 th week $(n=3)$, the protruding area was increased. The cover of the region with low density was stripped with strong and high signal; At 10th week $(n=5)$, signal of the cover upon the plaque were much thinner, while, pathology examination had proved that this signal represent the fibrous cap. Signal high bright region contain radial pattern that showed in the thin cap with lipid rich plaque was infiltrated with large macrophage; At 12th week $(n=4)$, OCT showed the lumen area was severely stenosis due to the large protrusion or ringed-shaped plaque formation. Fibrous cap thickness were less than $65 \mu \mathrm{m}$ (mean value $=0.038 \pm 0.01 \mathrm{~mm}$ ). While, $28.6 \%$ of the plaques were total occlusion lesion. Total Cholesterol (TC) was at 6 weeks $36.77 \pm 0.80 \mathrm{mmol} / 1$, 8 weeks $38.10 \pm 1.92 \mathrm{mmol} / \mathrm{l}$, 10 weeks $35.94 \pm 0.93 \mathrm{mmol} / \mathrm{l}, 12$ weeks $35.36 \pm 1.30 \mathrm{mmol} / \mathrm{l}$; Low density lipoprotein (LDL) was $26.79 \pm 4.64 \mathrm{mmol} / 1$ at 6 week, $20.72 \pm 2.07 \mathrm{mmol} / 1$ at 8 weeks, $19.83 \pm 1.96 \mathrm{mmol} / 1$ at 10 weeks, $19.83 \pm 1.96 \mathrm{mmol} / \mathrm{l}$ at 12 weeks.

Conclusion Balloon injury intima and subsequently high fat diet for 12 weeks can successfully establish the vulnerable plaque animal model. Plaques with large lipid core and thin cap were tend to rupture with subsequently thrombus. This process was the same as human beings, so that we can study the process of the vulnerable plaque formation using optical coherence tomography to display early stage imaging characteristics of the plaque. With the long time high fat diet, Total Cholesterol (TC) and Low Density Lipoprotein (LDL) had sharply increased which have a strong relativity with the stability of the plaque. Thus, we can use blood fat test to predict the stability changes of the plaque.

\section{e0242 CHANGES OF ADIPONECTIN EXPRESSION IN ACUTE MYOCARDIAL INFARCTION RATS AND THE SIGNIFICANCE OF BISOPROLOL INTERVENTION}

doi:10.1136/hrt.2010.208967.242

${ }^{1}$ Song Zhang, ${ }^{1}$ Baiming Qu, ${ }^{1}$ Lixuan Wu, ${ }^{2}$ Zuyue Wang. ${ }^{1}$ Department of Cardiovascular Diseases, People's Hospital, Zhejiang Province, China; ${ }^{2}$ Department of Cardiology, Washington Hospital Center, Washington, USA

Background and Aims The aim of this study was to explore the expression changes of myocardial APN in acute myocardial infarction (AMI) rats, changes of serum APN, and the significance of Bisoprolol intervention.

Methods AMI rat model was established for the purposes of this study and was used for analysis of serum APN as determined by ELISA. Expression changes of myocardial APN mRNA in AMI rats were determined via RT-PCR and expression changes of myocardial APN protein were determined by the way of immunohistochemistry. Results Serum APN concentration and APN protein expression of the myocardium decreased significantly in the AMI groups compared with the sham operation group, with the lowest serum APN and APN protein expression on day 7 after AMI. On days 7 and 10 after AMI, the expression of myocardial APN mRNA in the AMI groups decreased significantly compared with the sham operation group. However, the APN mRNA increased on day 10 compared with that on day 7. Notably, there was an increase in levels of serum APN and myocardial APN expression after Bisoprolol intervention. Conclusions The expression of myocardial APN and serum APN decreased in AMI rats. APN may be an important protective factor against AMI. Bisoprolol can also protect against AMI due to its increasing APN expression.

\section{Epidemiology and Preventive Medicine: Epidemiology of Cardiovascular Disease e0243 A STUDY ON EPIDEMIOLOGICAL TREND OF ELDERLY HYPERTENSION IN BEIJING}

doi:10.1136/hrt.2010.208967.243

ALi-Na, Tang Zhe, Zhao Xiao-Ling, Guan Shao-Chen, Sun Fei, Liu Hong-Jun, Wu XiaoGuang, Diao Li-Jun. Capital University of Medical Science, Beijing, China

Object To explore the epidemiological trend of elderly hypertension, so as to provide basis for prevention of hypertension. 